\title{
Measuring the effect of demagnetization in stacks of gadolinium plates using the magnetocaloric effect
}

\author{
Lipsø, Hans Kasper Wigh; Nielsen, Kaspar Kirstein; Christensen, Dennis ; Bahl, Christian Robert \\ Haffenden; Engelbrecht, Kurt; Kuhn, Luise Theil; Smith, Anders
}

\section{Published in:}

Journal of Magnetism and Magnetic Materials

Link to article, DOI:

10.1016/j.jmmm.2011.06.052

Publication date:

2011

Link back to DTU Orbit

Citation (APA):

Lipsø, H. K. W., Nielsen, K. K., Christensen, D., Bahl, C. R. H., Engelbrecht, K., Kuhn, L. T., \& Smith, A. (2011). Measuring the effect of demagnetization in stacks of gadolinium plates using the magnetocaloric effect. Journal of Magnetism and Magnetic Materials, 323(23), 3027-3032. https://doi.org/10.1016/j.jmmm.2011.06.052

\section{General rights}

Copyright and moral rights for the publications made accessible in the public portal are retained by the authors and/or other copyright owners and it is a condition of accessing publications that users recognise and abide by the legal requirements associated with these rights.

- Users may download and print one copy of any publication from the public portal for the purpose of private study or research.

- You may not further distribute the material or use it for any profit-making activity or commercial gain

- You may freely distribute the URL identifying the publication in the public portal 


\title{
Measuring the effect of demagnetization in stacks of gadolinium plates using the magnetocaloric effect
}

\author{
K.W. Lips $\emptyset^{1}$, K.K. Nielsen ${ }^{2,3, *}$, D.V. Christensen ${ }^{2,4}$, C.R.H. Bahl ${ }^{2}$, \\ K. Engelbrecht ${ }^{2}$, L.Theil Kuhn ${ }^{2}$ and A. Smith ${ }^{2}$ \\ 1 Department of Physics, Technical University of Denmark \\ DK-2800 Kgs. Lyngby, Denmark \\ ${ }^{2}$ Fuel Cells and Solid State Chemistry Division \\ Risø National Laboratory for Sustainable Energy, Technical University of Denmark \\ Frederiksborgvej 399, DK-4000 Roskilde, Denmark \\ ${ }^{3}$ Department of Mechanical Engineering, Technical University of Denmark \\ Building 425, Niels Koppels Alle, DK-2800 Kgs. Lyngby, Denmark \\ ${ }^{4}$ Niels Bohr Institute, University of Copenhagen \\ Blegdamsvej 17, DK-2100 Copenhagen, Denmark \\ * Corresponding author: kaki@risoe.dtu.dk
}

(Dated: March 11, 2011)

\begin{abstract}
The effect of demagnetization in a stack of gadolinium plates is determined experimentally by using spatially resolved measurements of the adiabatic temperature change due to the magnetocaloric effect. The number of plates in the stack, the spacing between them and the position of the plate on which the temperature is measured are varied. The orientation of the magnetic field is also varied. The measurements are compared to a magnetostatic model previously described. The results show that the magnetocaloric effect, due to the change in the internal field, is sensitive to the stack configuration and the orientation of the applied field. This may have significant implications for the construction of a magnetic cooling device.
\end{abstract}

PACS numbers: $75.30 . \mathrm{Sg}, 07.55 . \mathrm{Ge}, 75.50 . \mathrm{Cc}, 75.60 . \mathrm{Ej}, 41.20 . \mathrm{Gz}$ 


\section{INTRODUCTION}

Magnetic materials exhibit the magnetocaloric effect (MCE), which manifests itself as a change in temperature, $\Delta T_{\mathrm{ad}}$, when changing the magnetic field applied to the material under adiabatic conditions.

The MCE has been used since the 1920s as a tool to reach temperatures close to absolute zero. ${ }^{1}$ It was later suggested to utilize it at near-room temperature by using thermal regeneration to increase the temperature span in a so-called active magnetic regenerator (AMR). ${ }^{2,3}$ In Refs. 4-6 extensive reviews of the application of the MCE in AMR devices are given.

One of the main components of a magnetic refrigerator based on the AMR is a regenerator made of a porous magnetic material in thermal contact with a heat transfer fluid. This component acts as a thermal regenerator that stores/releases heat and supports a temperature gradient in the flow direction, thus upholding a cold and a hot end, in close interaction with the heat transfer fluid (which is typically aqueous for room temperature applications). While working as a regenerator the AMR is exposed to a periodic change in applied magnetic field, $H_{\text {appl }}$. In this way the MCE in terms of the adiabatic temperature change provides the active work input to the refrigeration cycle. ${ }^{3}$

The geometry of the regenerator may vary and typically either packed spheres ${ }^{7,8}$ or parallel plates ${ }^{9,10}$ are used. When numerical models of the AMR are considered the magnetic field is most often considered to be equal to the applied field, $H_{\text {appl }} \cdot{ }^{11-16}$ Recent material studies on a single rectangular plate subjected to conditions relevant for magnetic refrigeration show, however, that the internal magnetic field can differ significantly from the applied magnetic field. ${ }^{17,18}$ This is due to the demagnetizing field created by the magnetization of the regenerator. This field is a function of the magnetization and the geometry of the regenerator. ${ }^{17,19-21}$ The magnetization is in itself a function of the local field and temperature and given that the AMR is operating around the magnetic transition temperature of the material ${ }^{22}$, which is where the largest MCE is achieved, the magnetization is generally far from being homogeneous in the material.

In this paper we consider stacks of parallel plates where the plates are identical and made of gadolinium. The resulting internal magnetic field of such a stack is found using a magnetostatic model previously published. ${ }^{17}$ Experimentally, the adiabatic temperature change is measured directly on the surface of a single plate situated in various stack and 
magnetic field configurations.

\section{MAGNETOSTATIC DEMAGNETIZATION MODEL}

It is well known that a magnetized body generates a magnetic field that, inside the body, tends to oppose the applied field. When the body is homogeneously magnetized this demagnetizing field may be expressed through a demagnetization tensor field, $\mathbb{N}(\mathbf{r})$, in the following way

$$
\mathbf{H}_{\mathrm{dem}}(\mathbf{r})=-\mathbb{N}(\mathbf{r}) \cdot \mathbf{M}
$$

where $\mathbf{H}_{\mathrm{dem}}$ is the demagnetizing field and $\mathbf{M}$ is the magnetization. In general, $\mathbb{N}$ is a function of the shape of the magnetic body. For certain geometries, such as ellipsoids ${ }^{23}$, infinite sheets, cylinders ${ }^{24}$ and rectangular prisms $^{25}$ it may be found analytically.

When the magnetic body is not homogeneously magnetized, which is the case if, e.g., a temperature profile is present or the magnitude of $\mathbf{M}$ depends on the internal field, $\mathbf{H}$, Eq. 1 is not valid. The problem of finding the internal magnetic field given by

$$
\mathbf{H}=\mathbf{H}_{\mathrm{appl}}+\mathbf{H}_{\mathrm{dem}},
$$

is then coupled with finding the magnetization, which, in turn, is a function of the local field and temperature.

In Refs. 17,18 a numerical model of the demagnetizing field of rectangular prisms is presented. The model assumes a discretization into small rectangular sub-prisms, where the magnetization, internal field and temperature inside each sub-prism are assumed constant and homogeneous. In this way the analytical solution to Eq. 1 may be applied to each individual sub-prism and the solution of the entire system is then a superposition of the individual solutions. This is formulated mathematically as

$$
\mathbf{H}_{\mathrm{dem}}(\mathbf{r}) \approx-\sum_{i=1}^{N} \mathbb{N}\left(\mathbf{r}-\mathbf{r}_{i}^{\prime}\right) \cdot \mathbf{M}_{0}\left(\mathbf{H}\left(\mathbf{r}_{i}^{\prime}, T_{i}\right), \mathbf{r}_{i}^{\prime}, T_{i}\right),
$$

where $\mathbf{r}$ and $\mathbf{r}_{i}^{\prime}$ are the position vectors of the point at which the demagnetizing field is evaluated and the point contributing with the magnetization, $\mathbf{M}_{0}$, respectively. The index $i$ denotes the respective sub-prism, or grid cell, and $N$ denotes the number of grid cells. In this way the sum in Eq. 3 is taken over all the contributions to the resulting demagnetizing 


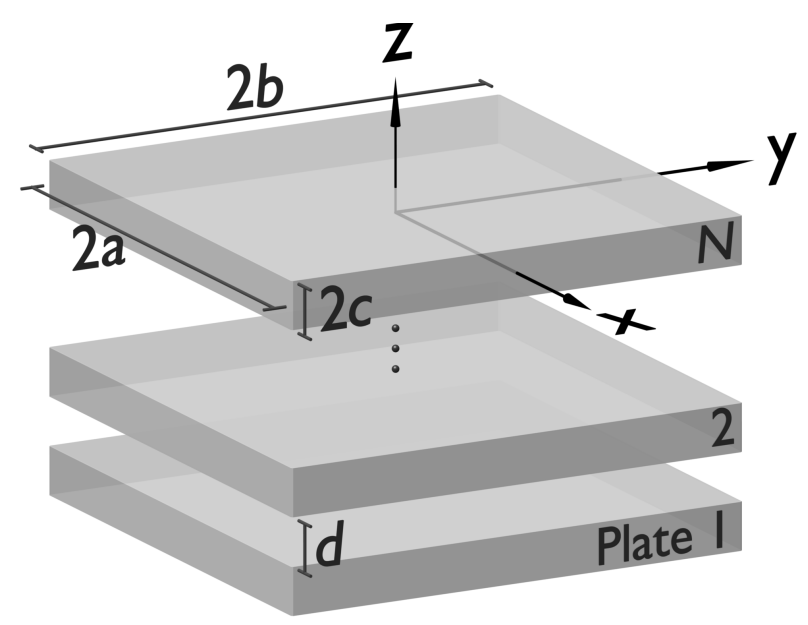

FIG. 1: The coordinate system of the stack of rectangular plates. The dimensions of each plate, $2 a \times 2 b \times 2 c$, are indicated in the figure as are the number of plates, $N$, and the distance between adjacent plates, $d$.

field at the location $\mathbf{r}$. The components of $\mathbb{N}(\mathbf{r})$ may be found in Ref. 17. Eqs. 2 and 3 are combined with an appropriate state function for $M(T, H)$ and the direction of the magnetization is assumed to be along $\mathbf{H} .{ }^{19}$ The model is then solved through iteration until it converges; see Ref. 17 for further details.

Magnetization data is that of commercial grade Gd and are taken from the experimental determination of the magnetization as a function of internal magnetic field and temperature published in Ref. 26. The adiabatic temperature change is found through interpolation from a table where it is a function of the internal magnetic field and temperature also published in Ref. 26.

The coordinate system employed for the stack of rectangular prisms is indicated in Fig. 1. The distance between the prisms is assumed constant and the prisms are assumed flat and uniform, i.e. plates. The distance between two adjacent plates is denoted $d$ and the thickness of a single plate is $2 c$. The stacking of rectangular plates will, in the case of magnetic refrigeration considered as an application, be as depicted in Fig. 1. Considering a single plate, application of a field along the $x$ - or $y$-direction will thus maximize the internal field due to the resulting minimized demagnetizing field. ${ }^{17}$ Application of the field along the $z$-direction will maximize the demagnetizing field and thus decrease the resulting internal field in a single rectangular plate. It follows qualitatively from Fig. 2 that application of the field along the $x$ - or $y$-direction will tend to create an opposing magnetic field outside the 


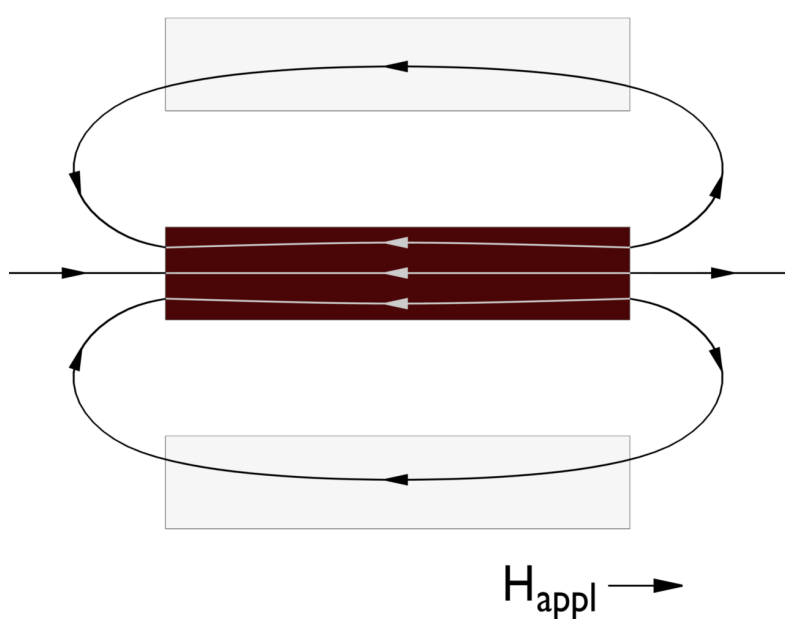

(a)



(b)

FIG. 2: Illustration of the magnetic field resulting from the magnetization of a single rectangular plate. In (a) the field is along the $x$-direction and thus parallel to the largest face of the plate. The resulting stray- or interaction field tends to oppose the applied field in the adjacent plates. In (b) the field is along the $z$-direction. The demagnetizing field inside the body is larger than in (a), however, the stray field tends to enhance the applied field in adjacent plates.

individual plates, thus lowering the field in neighboring plates. In the case of magnetizing along the $z$-direction, the field external to the individual plate will tend to align with the internal field of neighboring plates thus increasing their total internal magnetic field.

It may therefore be concluded that it is not a priori obvious which configuration is optimal. This must be expected to be dependent on the number of plates in the stack, their relative dimensions and their spacing. It is thus of importance to investigate this in detail, which is the topic of the remainder of this paper.

\section{EXPERIMENTAL}

A plastic housing is used to make the different stack configurations. 13 grooves of $1 \mathrm{~mm}$ have been machined with a spacing of $0.8 \mathrm{~mm}$. In this way various combinations of the number of plates and their positioning may be used.

The stacks are situated in and controlled by a device built at Ris $\emptyset$ National Laboratory for Sustainable Energy, Technical University of Denmark. ${ }^{9,28,29}$ The magnetic field is generated by a cylindrical Halbach magnet assembly, see Fig. 3, which attains its maximum of $1.1 \mathrm{~T}$ 


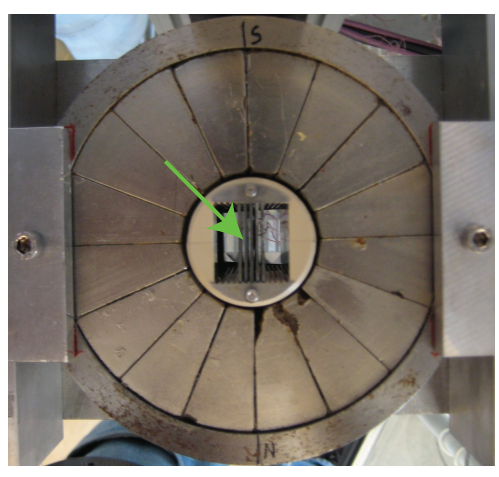

(a)

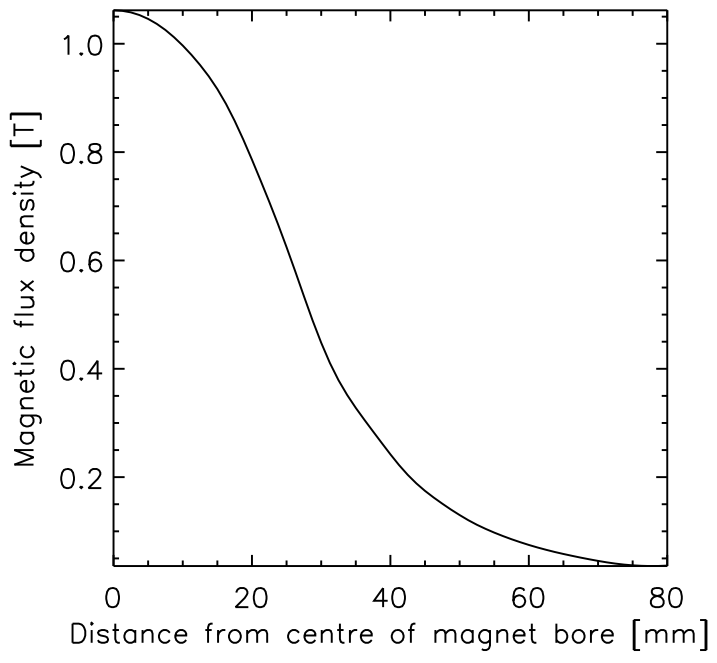

(b)

FIG. 3: (a) Plates (marked by arrow) and plastic cylinder fixed in the center of the Halbach magnet. As evident from the picture some of the magnet segments are damaged, resulting in a slightly asymmetric field. (b) the average magnetic flux density as a function of the distance along the rotational symmetry axis from the centre of the bore of the Halbach magnet (from Ref. 27).

in the center of the magnet; see Ref. 27 for details. When the stack has attained thermal equilibrium in the center of the magnetic field it is pulled out sufficiently fast to be considered adiabatic. The temperature is logged from five positions on one plate, see Fig. 4, with a TC-08 Thermocouple Data Logger from Pico Technology using Type E thermocouples with a sampling rate of $10 \mathrm{~Hz}$ per thermocouple. The thermocouples are attached to the $\mathrm{Gd}$ plate using a thermal paste (Loctite 315), which is electrically insulating; see Fig. 4. It is estimated that the sampling interval and the displacement time are significantly smaller than the time it takes the heat to dissipate. ${ }^{18}$

The experiments are conducted at $295 \mathrm{~K}$. This is close to the Curie temperature of gadolinium $\left(T_{\mathrm{C}}=293 \mathrm{~K}\right)$ and thus to the temperature at which the cooling device is expected to function. The gadolinium plates have the dimensions $40 \times 25 \times 0.9 \mathrm{~mm}^{3}$. They are obtained from China Rare Metal Material Co. with a stated purity of $99.9 \%$.

Each experiment is repeated five times and the average of the measurements is reported. The maximum observed standard deviation of the average values was $0.05 \mathrm{~K}$. The adiabatic temperature change predicted by the model is found by considering the corresponding loca- 


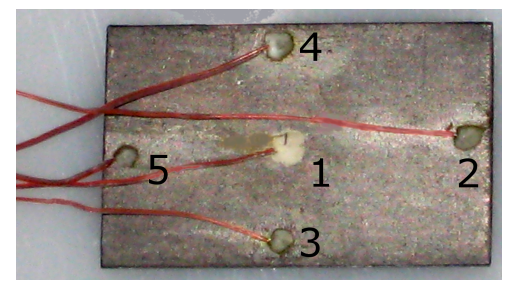

FIG. 4: Gadolinium plate with thermocouples mounted in the center and on the four edges.

tions of the thermocouples on the plate in the model. The applied field used in the model is given in Fig. 3(b).

\section{RESULTS AND DISCUSSION}

The experimental results are divided into three groups. Firstly, the number of plates in a stack is varied and the effect on the centre plate is analyzed. Secondly, the number of plates is held constant and the position in the stack of the plate on which the measurements are taken is varied. Finally, the influence of the packing density is investigated by keeping the height of the stack fixed, while the number of equally spaced plates and the distance between them are varied.

\section{A. Variation of the number of plates}

The adiabatic temperature change as a function of the number of plates is given in Fig. 5 for the centre plate equipped with thermocouples as depicted in Fig. 4. Considering Fig. 5(a), where the applied field is along the $z$-direction, the trend is clear. As the number of plates increases the observed magnetocaloric effect also increases. This is clearly observed both experimentally and predicted by the model. Qualitatively it is also to be expected since adding plates to a stack of rectangular plates where the applied field is along the direction of the stacking tends to increase the resulting field in adjacent plates due to the magnetization of each individual plate (see Fig. 2).

The adiabatic temperature change is also seen to be non-uniform across the plate since the thermocouples consistently report different temperature changes, although with a maximum difference across the plate for a given setup of approximately $0.3 \mathrm{~K}$. This is consistent with the fully spatially resolved adiabatic temperature change data obtained by demagnetizing 


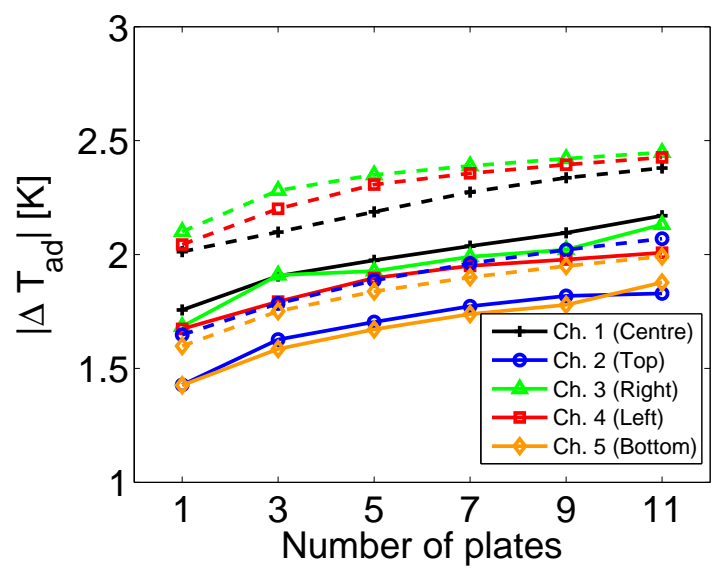

(a)

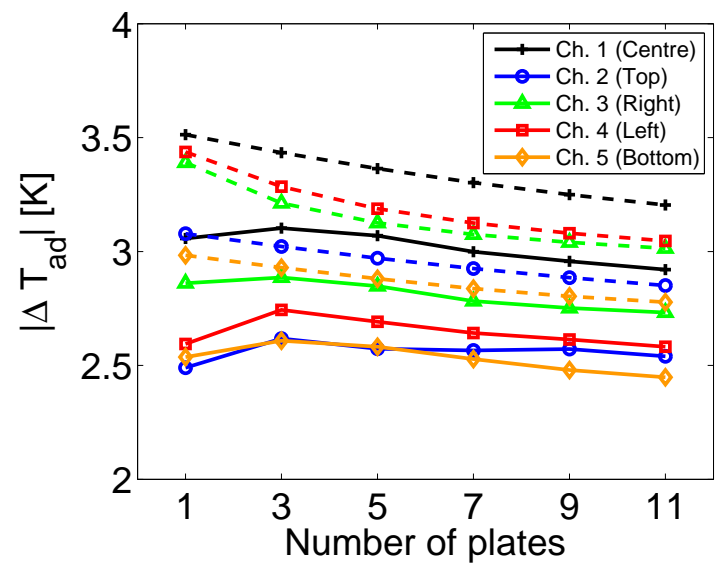

(b)

FIG. 5: The adiabatic temperature change of the centre gadolinium plate as a function of the number of plates in the stack. (a) the applied field is along the $z$-direction, i.e. orthogonal to the $40 \times 25 \mathrm{~mm}^{2}$ face and thus along the direction of the stacking. (b) the applied field is along the $x$-direction, i.e. parallel to the $40 \times 25 \mathrm{~mm}^{2}$ face. The dashed lines show the values predicted by the model.

a single plate of gadolinium recently reported in Ref. 18 under identical conditions. The reason for this behavior is a combination of that the applied field is not constant along the entire $40 \mathrm{~mm}$ direction of the plate (see Fig. 3(b) for clarity) and that the demagnetizing field is not homogeneous even if the plate was uniformly magnetized. ${ }^{17,19}$

Considering Fig. 5(b) it is observed that the trend of the magnetocaloric effect differs from that observed in Fig. 5(a). As the number of plates increases the resulting adiabatic temperature change tends to decrease. This is clear both from the model and the experimental data as well as it is expected from the more qualitative argument given in Sec. II. When the applied field is oriented orthogonally to the direction of the stacking, the field generated externally to each individually magnetized plate will tend to oppose the internal field in adjacent plates thus lowering the resulting internal field. The greatest difference in the observed MCE when considering a single plate and a stack of 11 plates is observed at the right thermocouple (green) where an adiabatic temperature change of approximately 3.4 $\mathrm{K}$ is lowered to approximately $3 \mathrm{~K}$, thus representing a relative difference of about $12 \%$.

In general, it should be noted that since the positions of the thermocouples (see Fig. 4) are not completely symmetric, both the model and the experiment are expected to deviate 


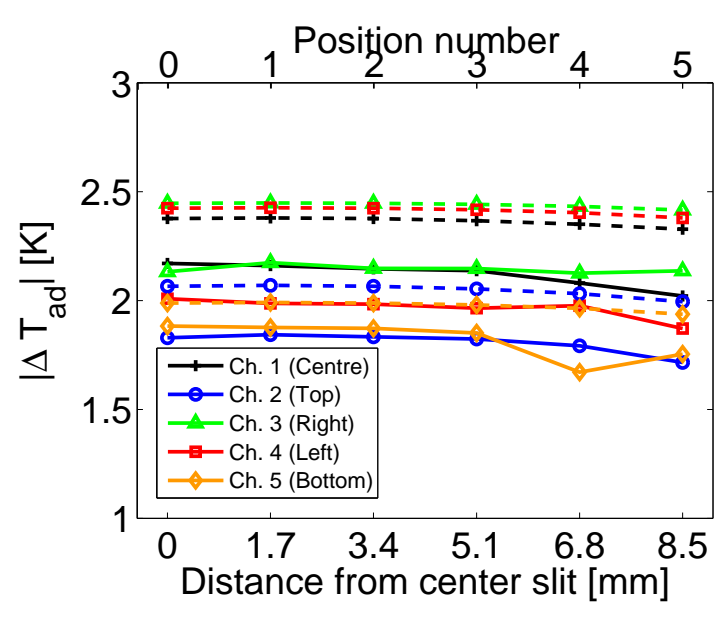

(a)

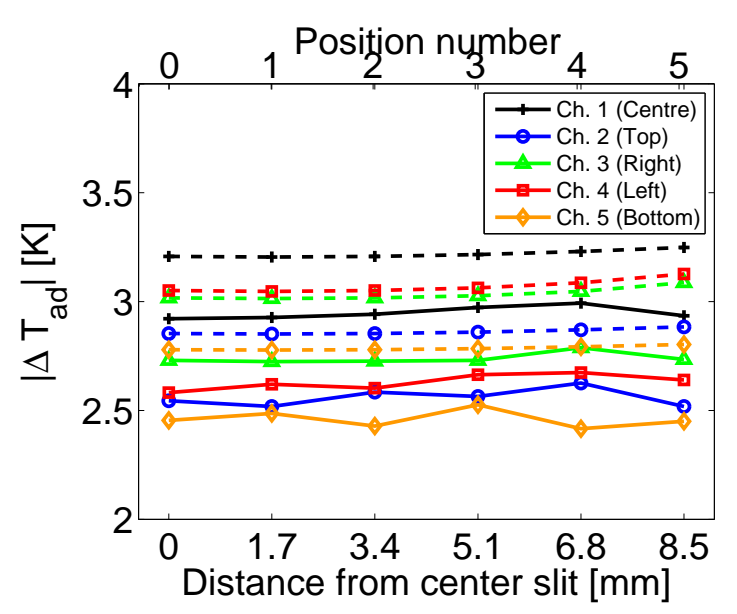

(b)

FIG. 6: The adiabatic temperature change when demagnetizing a stack of gadolinium plates as a function of position of a plate in a stack of 11 evenly spaced plates. (a) the applied field is along the $z$-direction, i.e. orthogonal to the $40 \times 25 \mathrm{~mm}^{2}$ face. (b) the applied field is along the $x$-direction, i.e. parallel to the $40 \times 25 \mathrm{~mm}^{2}$ face. The dashed lines show the corresponding values predicted by the model.

slightly. The top and bottom thermocouples are located almost identically on either side of the plate and should therefore, in principle, be exposed to the same change in applied magnetic field. The same is valid for the left and right thermocouples. However, small inhomogeneities in the applied field and uncertainties in the positioning of the thermocouples result in slightly deviating results.

Depending on the number of plates in the stack and the orientation of the applied field the difference in adiabatic temperature change across the plate is observed experimentally to be ranging approximately from 0.3 to $0.6 \mathrm{~K}$ equivalent to relative differences of 20 to 30 $\%$, respectively. This is a substantial variation that is supported by the data reported in Ref. 18.

\section{B. Variation of the position in the stack}

The adiabatic temperature change as a function of position in a stack of 11 identical plates is given in Fig. 6. The trends from both the model and experiment are the same. Considering the centre position going outwards until the second to last position the adiabatic temperature 


\begin{tabular}{l|ccc} 
Number of plates & 5 & 7 & 13 \\
\hline Spacing between plates $[\mathrm{mm}]$ & 4.2 & 2.5 & 0.8 \\
\hline Porosity & 0.79 & 0.70 & 0.45 \\
\hline Packing density & 0.21 & 0.30 & 0.55
\end{tabular}

TABLE I: Summary of the stack configurations used in order to probe the effect of packing density on the resulting magnetic field.

change is virtually constant in both cases and for both orientations of the applied field. At the outer position the magnetocaloric effect decreases slightly (about $0.1 \mathrm{~K}$ ) in the case where the field is applied along the $z$-direction, i.e. along the direction of the stacking. The tendency is a slight increase (about $0.1 \mathrm{~K}$ ) when the field is along the $x$-direction, i.e. parallel to the $40 \times 25 \mathrm{~mm}^{2}$ face. The changes are subtle and it is questionable whether the experimental data sufficiently support the trend. The model predicts a difference in the adiabatic temperature change of no more than $0.1 \mathrm{~K}$ in the most pronounced case.

Qualitatively, the predicted trend of the model is easily explained. In the case when the field is along the $z$-direction, the demagnetizing field is generally largest and on the outer plate the interaction with neighboring plates is minimized. Thus, the internal magnetic field is also minimized and the magnetocaloric effect is decreased. The opposite is the case when the applied field is along the $x$-direction. Here, the demagnetizing field is minimal on the outer plate, since the interaction from neighboring plates is small, and the magnetocaloric effect tends to increase.

\section{Influence of the packing density of the stack}

In order to probe the effect of varying spacing between adjacent plates in the stack, a stack with a fixed height of $21.3 \mathrm{~mm}$ is considered. One plate is fixed at the centre slit and the remaining plates are distributed in three different modes with 5, 7 and 13 plates, respectively. These modes correspond to uniform spacings between the plates of $4.2,2.5$ and $0.8 \mathrm{~mm}$, respectively. Table I gives a summary of the configurations.

When the applied field is oriented along the $z$-direction the magnetocaloric effect decreases as the spacing between adjacent plates increases whereas the opposite is true when the 


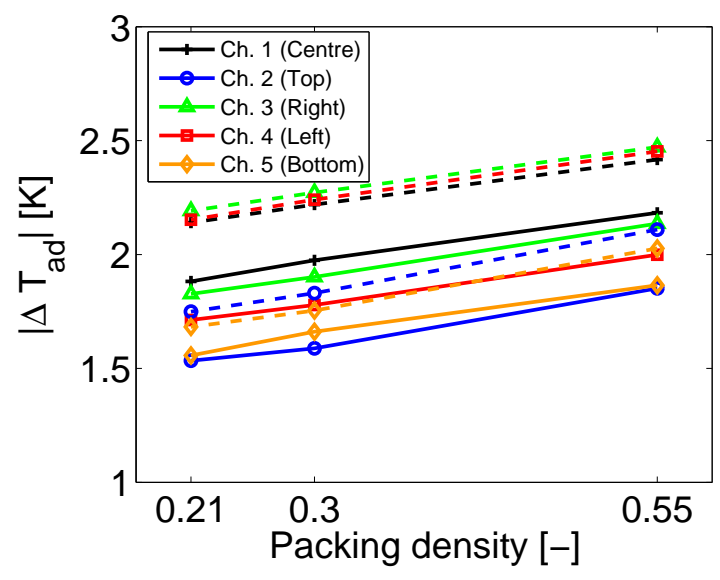

(a)

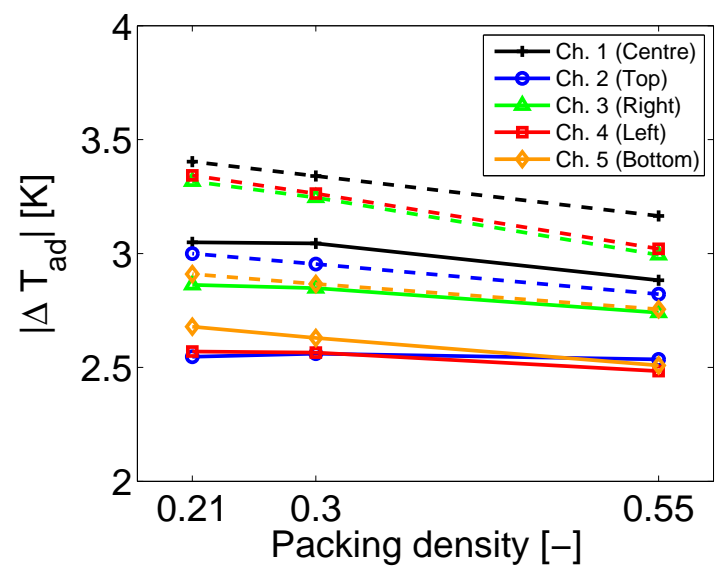

(b)

FIG. 7: The adiabatic temperature change when demagnetizing a stack of gadolinium plates as a function of the packing density. The height of the stack is constant (equal to $21.3 \mathrm{~mm}$ ). For a spacing of $0.8 \mathrm{~mm}$ the total number of plates is 13 , for a spacing of $2.5 \mathrm{~mm}$ it is 7 and finally for a spacing of $4.2 \mathrm{~mm}$ the number of plates is 5 . (a) the applied field is along the $z$-direction. (b) the applied field is along the $x$-direction. The dashed lines show the values predicted by the model.

applied field is along the $x$-direction. This is predicted both by the model and clearly observed experimentally; see Fig. 7. Furthermore, the adiabatic temperature change seems to decrease/increase linearly as a function of packing density, when the field is applied along the $x$ - and $z$-direction, respectively. This is an effect that is observed from both the model and the experimental data.

Considering the applied field along the $z$-direction and the spacing between adjacent plates to increase, the adiabatic temperature change and thus internal field decrease. Increasing the spacing between adjacent plates the stray field from adjacent plates will decrease. The situation may then be considered to approximate the single plate case and when the field is along the $z$-direction, this case yields the largest demagnetizing field with a smaller internal field as a result. The same explanation is valid for the case where the applied field is along the $x$-direction, however, here the effect is reversed since the single plate case results in a larger internal magnetic field. ${ }^{17}$ 


\section{Remarks on the experimental data}

All of the data points in Figs. 5-7 have small error bars, when these are considered as the standard deviation of the five measured values, i.e. the experiments are highly reproducable. The absolute experimental error has not been estimated. However, a few of the values differ from the overall trend. It was determined from model predictions that a deviation of $2 \mathrm{~K}$ in the surrounding temperature should result in a change in $\Delta T_{\mathrm{ad}}$ of less than $0.1 \mathrm{~K}$. A change of the same order of magnitude is predicted if the stack is misaligned a few degrees with respect to the orientation of the applied field.

Moreover, deviations can be caused by inhomogeneity in the applied field. As seen in Fig. 3 some of the magnetic blocks are damaged in the corners, causing small local perturbations of the field.

The experimental results have been compared to the model predictions in Figs. 5-7. An obvious correspondence between the behavior of the measured and the modeled data can be concluded. However, the adiabatic temperature change tends to be around $0.2 \mathrm{~K}$ smaller in the experiment than the model predicts. The discrepancy between model and experiment may be due to the heat capacity, heat transfer coefficient between the air and plate, or experimental uncertainty in the temperature measurement.

\section{CONCLUSIONS}

A significant change in the magnetocaloric effect expressed as the adiabatic temperature change when altering the magnetic field of a magnetocaloric material may be obtained depending on the overall geometry of the structure considered. In this study various stacks of identical plates of gadolinium were probed with attached thermocouples while the applied magnetic field was changed consistently. In the case of a single plate and applying the field along the largest face of the plate yields the largest internal magnetic field and thus the largest magnetocaloric effect. When the applied field is orthogonal to the largest face the internal field is minimized.

In this paper it was shown that when stacking identical plates the internal field is enhanced if the applied field is along the stacking direction, whereas stacking in a direction perpendicular to the applied field results in a decrease in the internal field. This was investi- 
gated using an experimental technique where the surface temperature change of gadolinium plates was measured and modeled through an established fully three-dimensional magnetostatic numerical model.

It is concluded that the results presented here are important to the continued development and understanding of the active magnetic regenerator (AMR). Only a limited amount of literature dealing with this system is concerned with the effect of demagnetization. ${ }^{17,20}$ It is an effect that may be crucial for any AMR geometry and it should thus be investigated further.

\section{Acknowledgements}

The authors thank the support of the Programme Commission on Energy and Environment (EnMi) (Contract no. 2104-06-0032) which is part of the Danish Council for Strategic Research. K.K. Nielsen also wishes to thank The Danish Council for Independent Research | Technology and Production Sciences (Contract no. 10-092791) for financial support. The technical assistance from J. Geyti, Risø DTU, is gratefully appreciated as are the input and suggestions from C. Frandsen, DTU Physics.

1 W. F. Giauque and D. P. MacDougall. Attainment of Temperatures Below $1 \mathrm{~K}$ Absolute by Demagnetization of $\mathrm{Gd}_{2}\left(\mathrm{SO}_{4}\right)_{3} \cdot 8 \mathrm{H}_{2} \mathrm{O}$. Physical Review, 43:768, 1933.

2 G. V. Brown. Magnetic heat pumping near room temperature. Journal of Applied Physics, 47(8):3673-3680, 1976.

3 J. A. Barclay. Theory of an active magnetic regenerative refrigerator. NASA Conference Publication, pages 375-387, 1983.

4 K.A. Gschneidner and V.K. Pecharsky. Thirty years of near room temperature magnetic cooling: Where we are today and future prospects. International Journal of Refrigeration, 31:945-961, 2008 .

${ }^{5}$ K. L. Engelbrecht, G. F. Nellis, S. A. Klein, and C. H. Zimm. Review article: Recent developments in room temperature active magnetic regenerative refrigeration. HVAC and R Research, 13(4):525, 2007. 
${ }^{6}$ K. K. Nielsen, J. Tusek, K. Engelbrecht, S. Schopfer, A. Kitanovski, C. R. H. Bahl, A. Smith, N. Pryds, and A. Poredos. Review on numerical modeling of active magnetic regenerators for room temperature applications. International Journal of Refrigeration, 34 (3):603-616, 2011.

7 C. Zimm, A. Boeder, J. Chell, A. Sternberg, A. Fujita, S. Fujieda, and K. Fukamichi. Design and performance of a permanent-magnet rotary refrigerator. International Journal of Refrigeration, 29(8):1302-1306, 2006.

8 A. Tura and A. Rowe. Progress in the characterization and optimization of a permanent magnet magnetic refrigerator. In P. W. Egolf, editor, Third International Conference on Magnetic Refrigeration at Room Temperature, pages 387-392. International Institute of Refrigeration, 2009 .

9 C. R. H. Bahl, T. F. Petersen, N. Pryds, and A. Smith. A versatile magnetic refrigeration test device. Review of Scientific Instruments, 79(9):093906, 2008.

10 P. A. Oliveira, P.V. Trevizoli, J. R. Barbosa Jr., and A. T. Prata. Numercial analsysis of a reciprocating active magnetic regenerator - part I. In P. W. Egolf, editor, Third International Conference on Magnetic Refrigeration at Room Temperature, pages 283-288. International Institute of Refrigeration, 2009.

11 K. Engelbrecht, G. F. Nellis, and S. A. Klein. Comparing modeling predictions to experimental data for active magnetic regenerative refrigeraton systems. In P. W. Egolf, editor, Second International Conference on Magnetic Refrigeration at Room Temperature, pages 349-357. International Institute of Refrigeration, 2007.

12 A. Sarlah and A. Poredos. Dimensionless numerical model for simulation of active magnetic regenerator refrigerator. International Journal of Refrigeration, 33 (6):1061-1067, 2010.

13 T. F. Petersen, N. Pryds, A. Smith, J. Hattel, H. Schmidt, and H.J.H Knudsen. Two-dimensional mathematical model of a reciprocating room-temperature active magnetic regenerator. International Journal of Refrigeration, 31:432-443, 2008.

14 K. K. Nielsen, C. R. H. Bahl, A. Smith, R. Bjørk, N. Pryds, and J. Hattel. Detailed numerical modeling of a linear parallel-plate active magnetic regenerator. International Journal of Refrigeration, 32(6):1478-1486, 2009.

15 K. K. Nielsen, C. R. H. Bahl, A. Smith, N. Pryds, and J. Hattel. A comprehensive parameter study of an active magnetic regenerator using a $2 \mathrm{~d}$ numerical model. International Journal of Refrigeration, 33(4):753-764, 2010. 
16 R. Bjørk and K. Engelbrecht. The influence of the magnetic field on the performance of an active magnetic regenerator (AMR). International Journal of Refrigeration, 34:192-203, 2011.

17 A. Smith, K. K. Nielsen, D. V. Christensen, C. R. H. Bahl, R. Bjørk, and J. Hattel. The demagnetizing field of a nonuniform rectangular prism. Journal of Applied Physics, 107(10):103910, 2010.

18 D. V. Christensen, R. Bjørk, K. K. Nielsen, C. R. H. Bahl, A. Smith, and S. Clausen. Spatially resolved measurements of the magnetocaloric effect and the local magnetic field using thermography. Journal of Applied Physics, 108(6):063913, 2010.

19 J.A. Brug and W.P. Wolf. Demagnetizing fields in magnetic measurements i. thin discs. Journal of Applied Physics, 57(10):4685-4694, 1985.

20 O. Peksoy and A. Rowe. Demagnetizing effects in active magnetic regenerators. Journal of Magnetism and Magnetic Materials, 288:424-432, 2005.

21 C. R. H. Bahl and K. K. Nielsen. The effect of demagnetization on the magnetocaloric properties of gadolinium. Journal of Applied Physics, 105(1):013916 (5 pp.), 2009.

22 A.M. Rowe and J.A. Barclay. Ideal magnetocaloric effect for active magnetic regenerators. Journal of Applied Physics, 93(3):1672-1676, 2003.

23 J.A. Osborn. Demagnetizing factors of the general ellipsoid. Physical Review, 67:351-357, 1945.

24 R.I. Joseph and E. Schloemann. Demagnetizing field in nonellipsoidal bodies. Journal of Applied Physics, 36(5):1579-1593, 1965.

25 A. Aharoni. Demagnetizing factors for rectangular ferromagnetic prisms. Journal of Applied Physics, 83(6):3432-3434, 1998.

26 R. Bjørk, C.R.H. Bahl, and M. Katter. Magnetocaloric properties of $\mathrm{LaFe}_{13-x-y} \mathrm{Co}_{x} \mathrm{Si}_{y}$ and commercial grade Gd. Journal of Magnetism and Magnetic Materials, 322(24):3882-3888, 2010.

27 K. K. Nielsen, R. Bjørk, J. B. Jensen, C. R. H Bahl, N. Pryds, A. Smith, A. Nordentoft, and J. Hattel. Magnetic Cooling at Risø DTU. In S. Hansen and J. Paul, editors, 8th IIR Gustav Lorentzen Conference on Natural Working Fluids. International Institute of Refrigeration, 2008.

28 K. Engelbrecht, J.B. Jensen, C.R.H. Bahl, and N. Pryds. Experiments on a modular magnetic refrigeration device. In P.W. Egolf, editor, 3rd International Conference on Magnetic Refrigeration at Room Temperature, pages 431-436. International Institute of Refrigeration, 2009.

29 K. Engelbrecht, C. R. H. Bahl, and K. K. Nielsen. Experimental results for a magnetic refrigerator using three different types of magnetocaloric material regenerators. International Journal 
of Refrigeration, Accepted, 2011. 